\title{
Decline in Immunization Coverage Across Well-performing Districts in India: An Urban Conundrum? Correspondence
}

\author{
Pinky Bahl • Jacob Puliyel
}

Received: 28 October 2014 / Accepted: 1 December 2014 /Published online: 16 December 2014

(C) Dr. K C Chaudhuri Foundation 2014

To the Editor: Dasgupta and colleagues have reported on the decline in immunization coverage in India and tried to analyze the factors responsible for it in states where coverage was good formerly [1]. They have found that areas with poor access to public health centre (PHC) and areas with poor people had a greater decline in immunization rates.

The explanatory variables they have selected are taken from DLHS3 (2007-2008). They have not shown that there is a change in these variables between DLHS2 and DLHS3 which corresponds to the change in immunization rates seen. If the number of PHC has declined in an area and there is a corresponding fall in immunization, their hypothesis would have been acceptable. I feel the authors have not investigated the phenomena adequately. Infrastructure problems result in low immunization uptake. The authors need to show that infrastructure has declined in states which had good coverage in the past, to prove their point.

The authors speculate whether reports of adverse events and deaths following immunization with pentavalent vaccine and factors related to the polio eradication campaign could be alternate reasons for the decline in immunization uptake. DLHS 3 was done in 2007-2008, when pentavalent vaccine was not even introduced in universal immunization program (UIP). So this is unlikely to be the cause of this decline.
It is a fact that the focus on pulse polio could have affected routine immunization adversely. The house to house visits for pulse -polio immunization and the 'just two drops' polio propaganda could have mislead people to think that polio is the only important vaccine needed for their children. This could have resulted in a decline in the enthusiasm for routine immunization where they have to go to health centers repeatedly.

By the time their article was published, the DLHS 20122013 results have become available [2]. There is a $25 \%$ decline in full immunization in Tamil Nadu between 2008 and 2012. Immunization coverage in TN in 2012 is only $56 \%$. This needs a more serious and in-depth analysis then what is provided in this paper.

Conflict of Interest None.

Source of Funding None.

\section{References}

1. Dasgupta R, Dasgupta P, Agrawal A. Decline in immunization coverage across well-performing districts in India: an urban conundrum? Indian J Pediatr. 2014;81:847-9.

2. Ministry of Health and Family Welfare. District level household survey 4 (2012-13). Available at https://nrhm-mis.nic.in/SitePages/ DLHS-4.aspx Accessed on 16 Oct.
P. Bahl $(\bowtie) \cdot J$. Puliyel

Department of Pediatrics, St. Stephens Hospital, Delhi 110054, India

e-mail: drpinkybahl@gmail.com 